\title{
A General Model of Hindered Diffusion
}

\author{
Shaltiel Eloul and Richard G. Compton* \\ Department of Chemistry, Physical \&6 Theoretical Chemistry Laboratory Oxford University, \\ South Parks Road, Oxford OX1 3QZ (UK) \\ E-mail: richard.compton@chem.ox.ac.uk \\ Phone: +44 (0)1865 275957. Fax: +44 (0) 1865275410
}




\begin{abstract}
The diffusion of a particle from bulk solution is slowed as it moves close to an adsorbing surface. A general model is reported which is easily applied by theoreticians and experimentalists. Specifically, it is shown here that in general and regardless of the space size, the magnitude of the effect of hindered diffusion on the flux is a property of the diffusion layer thickness. We quantify and explain the effect. Predictions of concentration profiles show that a 'hindered diffusion layer' is formed near the adsorbing surface within the diffusion layer, observed even when the particle radius is just a $0.1 \%$ of the diffusion layer thickness. In particular we focus on modern electrochemistry processes involving with impact of particles with either ultra-small electrodes or particles in convective systems. The concept of the 'hindered diffusion layer' is generally important for example in recent biophysical models of particles diffusion to small targets.
\end{abstract}

\title{
Graphical TOC Entry
}

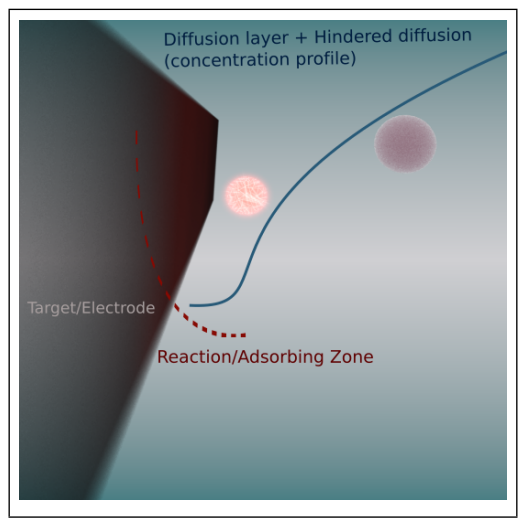


Predicting the events of particles stochastically hitting a surface target by virtue of their Brownian motion is important for understanding many biophysical and chemical processes. Modeling such events is especially a growing interest in modern electrochemistry, where reactions on electrodes can be utilised to detect individual particles impacting on electrodes. ${ }^{1-3}$ During the process, the particle diffuses from bulk solution towards a tunneling zone located at a short distance from electrode, where it may undergo a direct charge transfer reaction or may mediate a reaction between the electrode and a solution phase species. ${ }^{4,5}$ The impact analysis enables the study and detection of many types of particles such as metal nano-particles, ${ }^{6,7}$ bio-molecules ${ }^{8-10}$ including bacteria, ${ }^{11}$ and agglomerates. ${ }^{12,13}$

The transport of particles, from bulk solution to a reaction zone close to the surface of a target such as an electrode is usually described by Brownian motion, and can be modeled with the diffusion equation.

$$
\frac{\partial c}{\partial t}=\nabla \cdot(D \cdot \nabla c)
$$

In the bulk, particles have a characteristic diffusion coefficient $D_{0}$, reflecting their size. The Stokes-Einstein relation presents the bulk diffusion coefficient of a spherical particle in the limit of low Reynolds numbers, providing a good estimation for $D_{0}$ of particles even of only few nano-metres in size: ${ }^{14}$

$$
D_{0}=\frac{k_{B} T}{6 \pi \eta r_{p}}
$$

where $\eta$ is the dynamic viscosity, $k_{B}$ is the Boltzmann constant, and $T$ is the temperature. When particles diffuse close to the wall their hydrodynamic mobility is reduced and therefore their diffusion is hindered. Brenner ${ }^{15}$ has calculated the corrections to the Stokes-Einstein relation for the normal diffusion coefficients near a wall in the form of infinite series. Bevan and Prieve have accurately approximated the analytical solution made by Brenner for a sphere with a radius $r_{p}$ to the convenient form:

$$
D(h)=\frac{6 h^{2}+2 r_{p} h}{6 h^{2}+9 r_{p} h+2 r_{p}^{2}} \cdot D_{0}
$$


where $h$ is the separation distance between the wall and the closest point of the particle surface. The expression was also found to agree well with optical measurements for a ratio of separation $(h)$ to the particle radius, $h / r_{p}=40 \mathrm{~nm} / 3.5 \mu \mathrm{m} \sim 0.01 .^{16,17}$

In the literature, the effect of hindered diffusion is usually discussed in the context of confined spaces, and is therefore evident in porous media such as zeolites. ${ }^{18}$ It is also crucial in biological systems ${ }^{19,20}$ such as for predicting the delivery of nano-medicine in the confined zone of a tumour rich in collagen. ${ }^{21}$ In a non-confined space, when the particle diffuses from the bulk to a surface, this effect is usually and legitimately disregarded. This is due to the fact that the particle spends a negligible time close to a wall whilst searching the target for most of the time, far away from any wall. For instance, in nano-impact experiments, the observed mass transport of silver nano-particles showed a good agreement with free diffusion without any consideration of hindered diffusion. ${ }^{22,23}$

However, we have recently shown that the effect of hindered diffusion is important for calculating the steady-state flux or frequency of particles impact and the mean first passage time towards a small electrodes or generally adsorbing targets of the order of a micrometre. This effect is seen regardless of the diffusion space size $^{24}$ and is due to the convergent diffusion field which arises in small targets. This observation supports the calculation of 'impacts' towards a wire target as an electrode and explains both the high number of particle impacts detected despite the large size of the particles ${ }^{11}$ and the close fitting to pure Fickian diffusion with a bulk diffusion coefficient at a $5 \mu \mathrm{m}$ microdisc electrode. ${ }^{22}$

The effect of hindered diffusion is significant, because effectively, the search of the particle for the target is confined by the diffusion layer $\delta$ (A schematic example of a concentration profile in a Nernst layer towards an electrode as a target is shown in figure 1). In more detail, outside the diffusion layer, the probability of a particle, starting at position $x>\delta$ to find the electrode at time $t$, is uniform ( $c \sim c_{0}$, the bulk concentration), and hence the position of the particle is irrelevant for the searching time. Within the diffusion layer $x<\delta$, the position of the particle, becomes important and influences the searching time; the closer 
is the particle to the surface the shorter is the average time it takes for finding the target. Subsequently, the search is limited to only a short distance (within the diffusion layer) and thus the particle diffusion can be hindered for a significant fraction of the particle searching time and as function of the diffusion layer thickness.

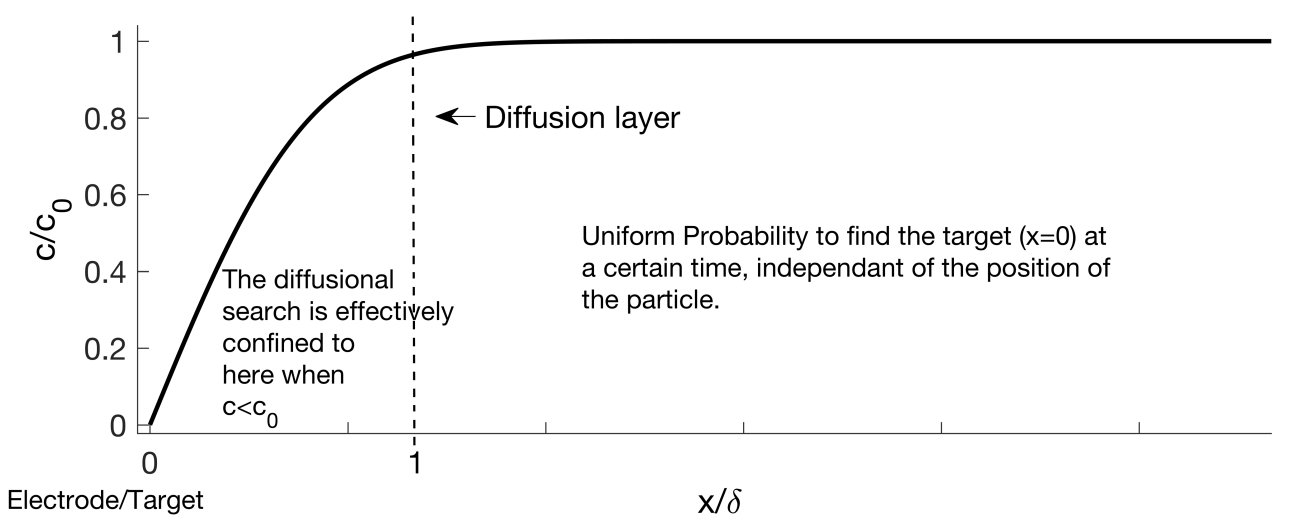

Figure 1: Illustration of a typical concentration profile along the cell (the surface is defined at $x=0$ ) for explaining the effect of hindered diffusion as function of the diffusion layer thickness.

The diffusion layer thickness, can be in many cases a short distance from the surface. In the context of small electrodes or adsorbing targets this is due to their typical convergent diffusion field which results in a steady diffusion layer of the order of the target size. ${ }^{24}$

Alternatively, a thin convergent diffusion layer can be observed by inclusion of a convection force. A typical example is the rotating disc electrode (RDE) where the diffusion layer is steady and its thickness can be in the order of a micrometre due to an additional velocity field normal to a rotating electode surface. ${ }^{25}$ The effect of hindered diffusion in RDE systems was also observed in a recent study. ${ }^{26}$

Moreover, the concept of the "Nernst diffusion layer" suggests that under non-forced hydrodynamic conditions, microscopic ("natural") convection in a cell causes the diffusion layer to be approximately finite and of the order of $0.01 \mathrm{~cm} .{ }^{25,27,28}$ Accordingly, the influence of hindered diffusion can be observed even for particles diffusion towards macro size electrodes/targets without considering any additional force. 
This letter is inspired by studies of electrochemical processes of particles which are optimal for capturing and understanding diffusion processes. It also has a broad significance in other diffusion problems of particle towards small targets. How viruses travel towards a nuclear pore, ${ }^{29}$ exit rate through an ultra small domain in synapse regulation process, ${ }^{30}$ and various nano-particle and macro-molecule attachment models; ${ }^{31,32}$ are all a few key models which rely on diffusion transport of particles towards a surface, and are particularly relevant for a fundamental study of hindered diffusion due to their convergent diffusion layer property as discussed above.

In this letter, we provide a general model that introduces the hindered diffusion effect on the flux of particles towards electrodes or adsorbing surfaces. For hindered diffusion modeling it is also necessary to consider the reaction zone/adsorbing layer, in order to capture correctly the effect the hindered diffusion. ${ }^{24,26}$ In the case of electrode, this is considered through a tunneling probability density function (discussed below) which describes the electron transfer reaction as function of distance from the electrode surface. Subsequently, the letter provides the calculations for the general effect of hindered diffusion layer on flux as function of the diffusion layer and also as function of the tunneling layer size.

For simplicity and clarity, a one dimensional diffusion problem is considered with an electrode or a surface at $x=0$. Therefore, the diffusion equation for particles with concentration, $c$ and including hindered diffusion becomes:

$$
0=\frac{\partial c(x, t)}{\partial t}=D(x) \frac{\partial^{2} c}{\partial x^{2}}+\frac{\partial D(x)}{\partial x} \frac{\partial c}{\partial x}-P
$$

$D(x)$ is the hindered diffusion coefficient calculated in the normal direction. A sink term is added for describing the adsorbing layer via a tunneling probability density function $P$. This models the impact of a particle as a fast reaction in the tunneling zone close the surface. The function follows an exponential fall off of the tunneling probability with distance:

$$
P=c(x) \nu \mathrm{e}^{-\beta x}
$$


$\nu$ is the tunneling rate $\left(s^{-1}\right)$ of the process. The exponential pre-factor can be varied. $\beta$ is the electron decay factor $\left(m^{-1}\right)$ which is solvent dependent and can be measured from scanning tunneling microscope measurements. It is thought to be $1.59 \AA^{-1}$ in aqueous media. ${ }^{33,34}$ The effect of $\beta$ dominates the adsorbing layer distance and is discussed in the analysis below.

Since we look for the effect of hindered diffusion in respect to a finite diffusion layer, a dimensionless analysis is carried out, where the $x$ coordinate is normalised to the thickness of the diffusion layer $\delta$ :

$$
X=x / \delta
$$

Accordingly, the particle radius is normalised to be:

$$
R_{p}=r_{p} / \delta
$$

and the diffusion coefficient and concentrations are:

$$
\begin{gathered}
D^{*}(X)=D(X) / D_{0} \\
C=c / c_{0}
\end{gathered}
$$

The normalisation results in the dimensionless form of the diffusion problem:

$$
0=D^{*}(X)\left(\frac{\partial^{2} C}{\partial X^{2}}\right)-P^{*}
$$

where the dimensionless sink term is given as:

$$
P^{*}=\frac{C \delta^{2}}{D_{0}} \nu \mathrm{e}^{-\beta^{*} X}
$$

and importantly $\beta^{*}=\beta \delta$.

The ordinary differential equation is solved with reflection boundaries condition at $X=0$, and at $X=1$ and the convergent diffusion layer is fulfilled with setting the concentration 
to $C=1$. The dimensionless flux towards the target is then calculated via integration of $P^{*}$ over all the space:

$$
\int_{0}^{1} P^{*} d X
$$

Assuming a diffusion layer of size $\delta$. Note that the flux can be return to dimensional units by:

$$
J_{d}=J \frac{D_{0} c_{0}}{\delta}
$$

Numerical calculations for solving the ODE was carried out using second order one-dimensional boundary value method with a non-uniform grid. The calculated dimensionless steady-state flux is calculated as function of $R_{p}$ and is shown in figure 2. When the dimensionless flux is

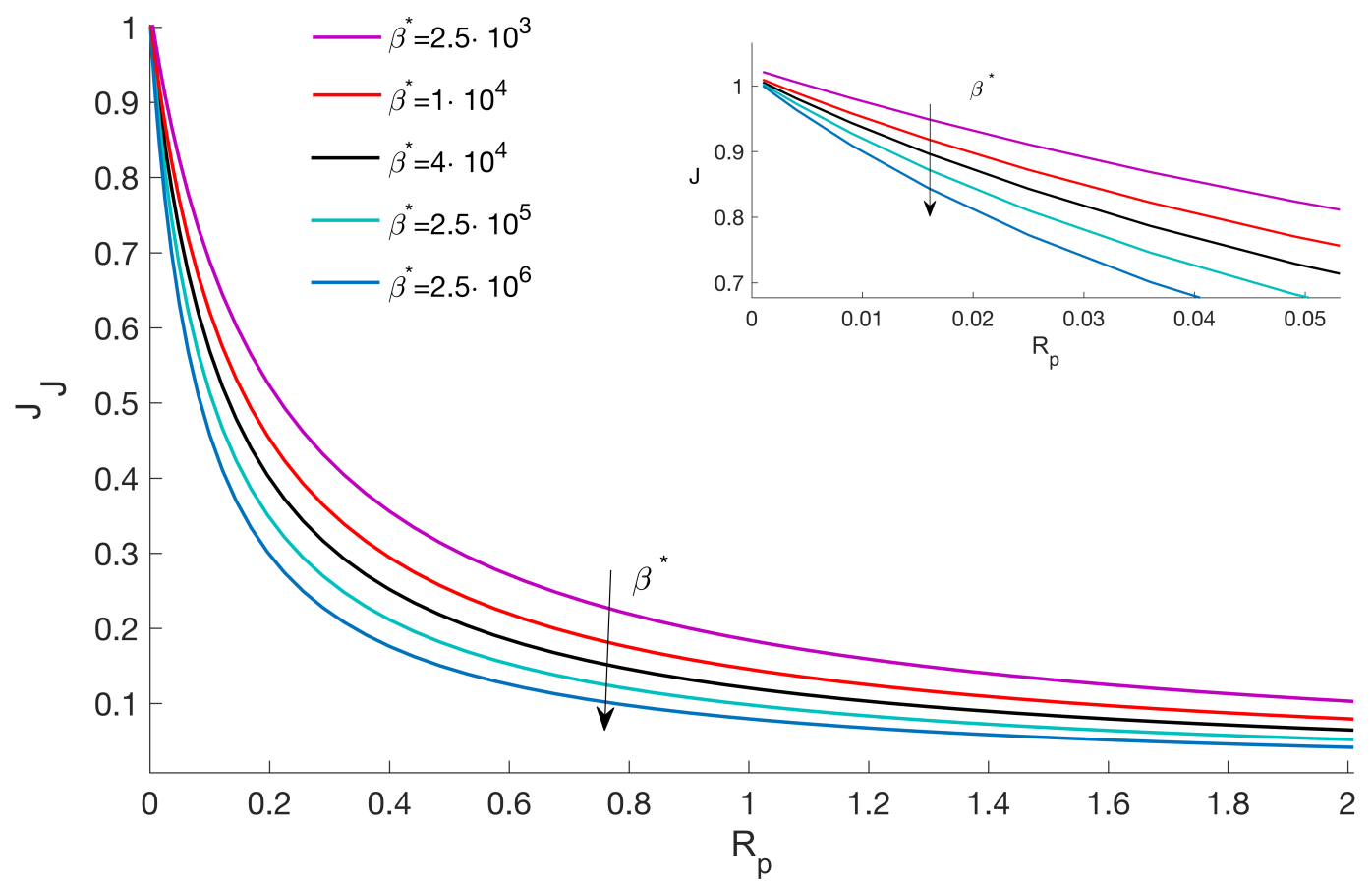

Figure 2: The dimensionless steady-state flux as function of $R_{p}$ and for various $\beta^{*}$. Then an inset shows a zoom view into small values of $R_{p}$.

close to unity the effect of hindered diffusion is negligible, and the flux can be approximated as:

$$
J \sim \frac{\Delta C}{\Delta X}
$$


It is worth mentioning that the above flux approximation is true only if the adsorbing layer is negligible in respect to the diffusion layer.

The strong effect of the size of the particle relative to the size of the diffusion layer $\left(R_{p}\right)$ is observed clearly in figure 2, providing the important conclusion that hindered diffusion becomes dominant as the convergent diffusion layer becomes small for a certain particle size. This explains why the flux of particles towards small electrodes, or towards a rotating disc will show a significant effect on the relative flux, due to the importance of the hindered diffusion, regardless of the extent of the bulk solution. Even when considering a Nernst diffusion layer, which can be of the order of $100 \mu \mathrm{m}$ in size, the effect of hindered diffusion may be seen for large particle sizes (micron-sized particles).

The effect of the adsorbing layer $\beta^{*}$ is also significant as can be seen for the various plots in figure 2. When $\beta^{*}$ becomes larger the exponential decay of the adsorbing layer shrinks to even shorter distances which requires the particles to diffuse even closer to the wall, before a reaction occurs. Note that $\beta^{*}$ is given as $\beta \delta$, which suggests that larger diffusion layers actually reduce the flux for an invariant $R_{p}$. It is also worth mentioning that for aqueous media $\left(\beta \sim 1.6 \cdot 10^{10}, \beta^{*}\right.$ has a large range of values. For instance, a Nernst diffusion layer of the order of $100 \mu \mathrm{m}$ leads to $\beta^{*}$ in the order of $10^{6}$, and for rotating disc electrode ${ }^{35}$ or submicro targets, ${ }^{36} \beta^{*}$ can be in the order of $10^{3}$.

The concentration profiles for various $R_{p}$ but a constant $\beta^{*}$ are plotted in figure 3 . The profiles shows the effect of the hindered diffusion on $\delta$ and reveals 'crowding' of the concentration as $R_{p}$ increases. The effect propagates through out the diffusion layer thickness $(\mathrm{X}=0$ to 1$)$.

We define the term 'hindered diffusion layer' as an appropriate description for a diffusion layer where $R_{p} \gg 0$.

Finally, the concentration profiles along $\mathrm{X}$ and as function of $\beta^{*}$ are compared for the values of $R_{p}=0.1$ and $R_{p}=0.001$ in figures 4 and 5 . The inset figures shows the concentration behaviour of the adsorbing layer very close to the surface. The results for various $\beta^{*}$ 


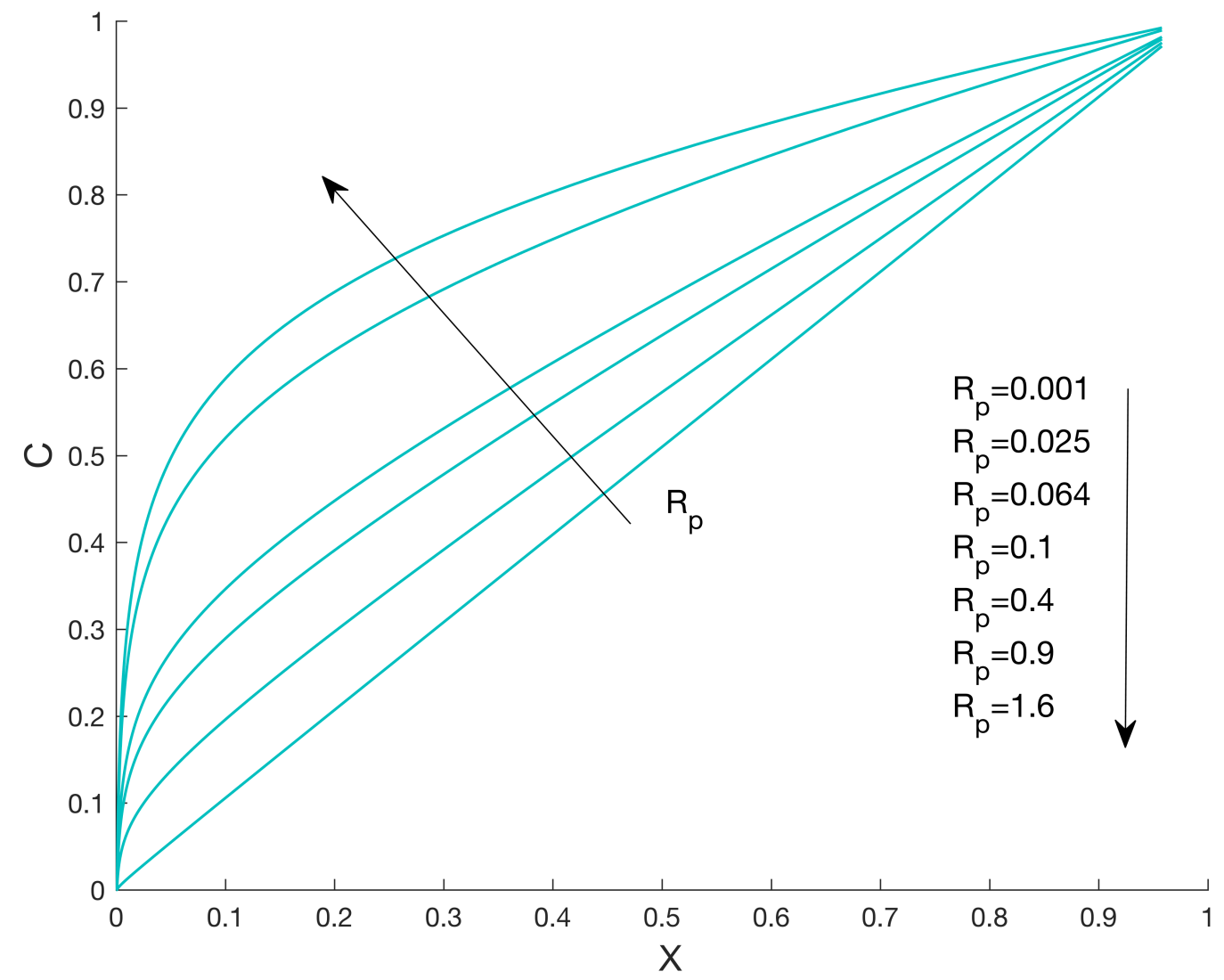

Figure 3: Concentration profiles as function of $\delta$ for various $R_{p} \cdot \beta^{*}=4 \cdot 10^{4}$. 
shows how the 'hindered diffusion layer' is highly influenced by the adsorbing zone through out the diffusion layer as observed for $R_{p}=0.1$ (4). The strong effect is due to the decrease of the diffusion coefficient as the adsorbing layer shrinks. In the case of $R_{p}=0.001$ (5), the change to the 'hindered diffusion layer' is small, however remains visible.

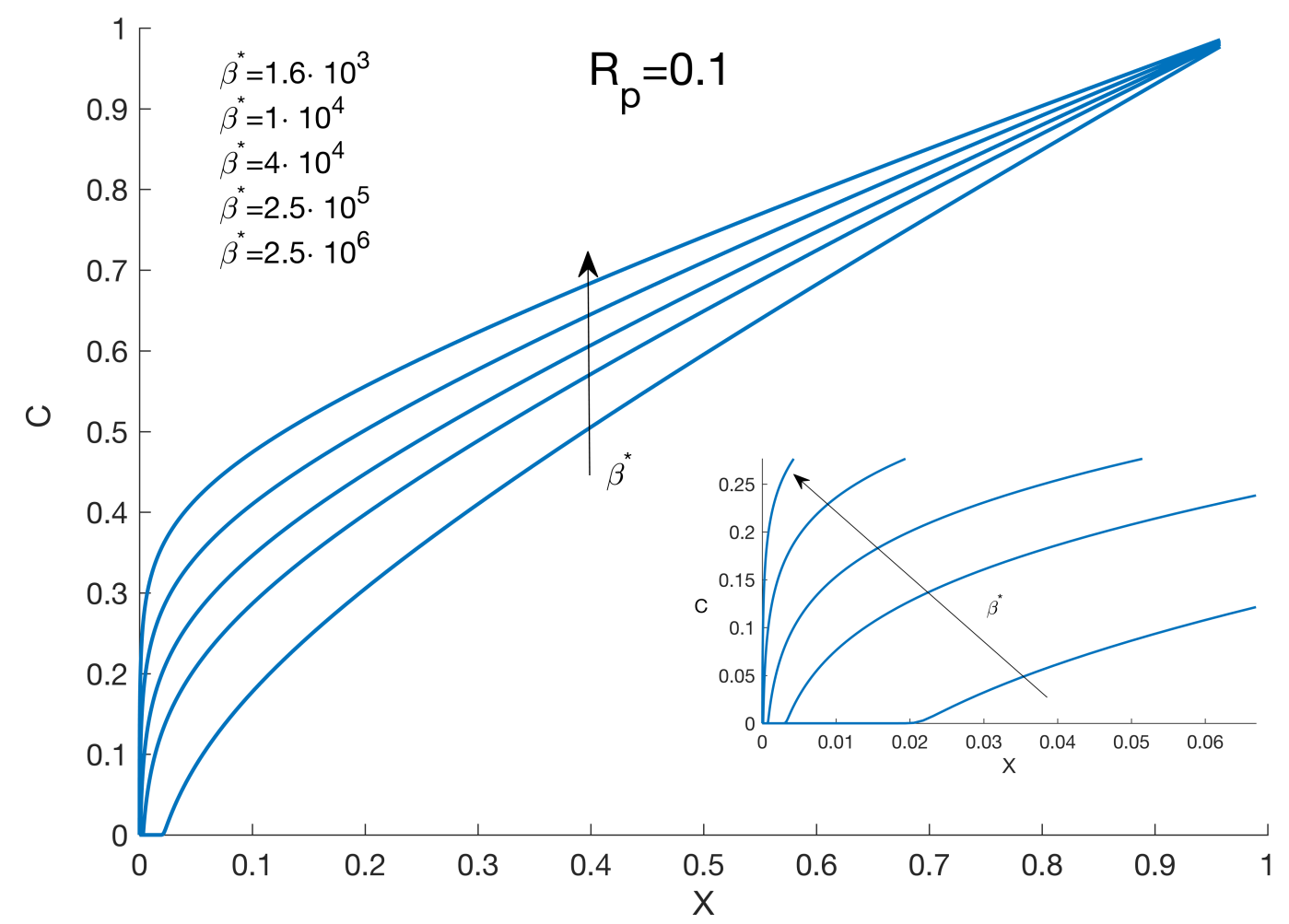

Figure 4: Concentration profiles as function of $\delta$ for various $\beta^{*} . R_{p}=0.1$. The inset shows a close view of the adsorbing layer next to the electrode. 


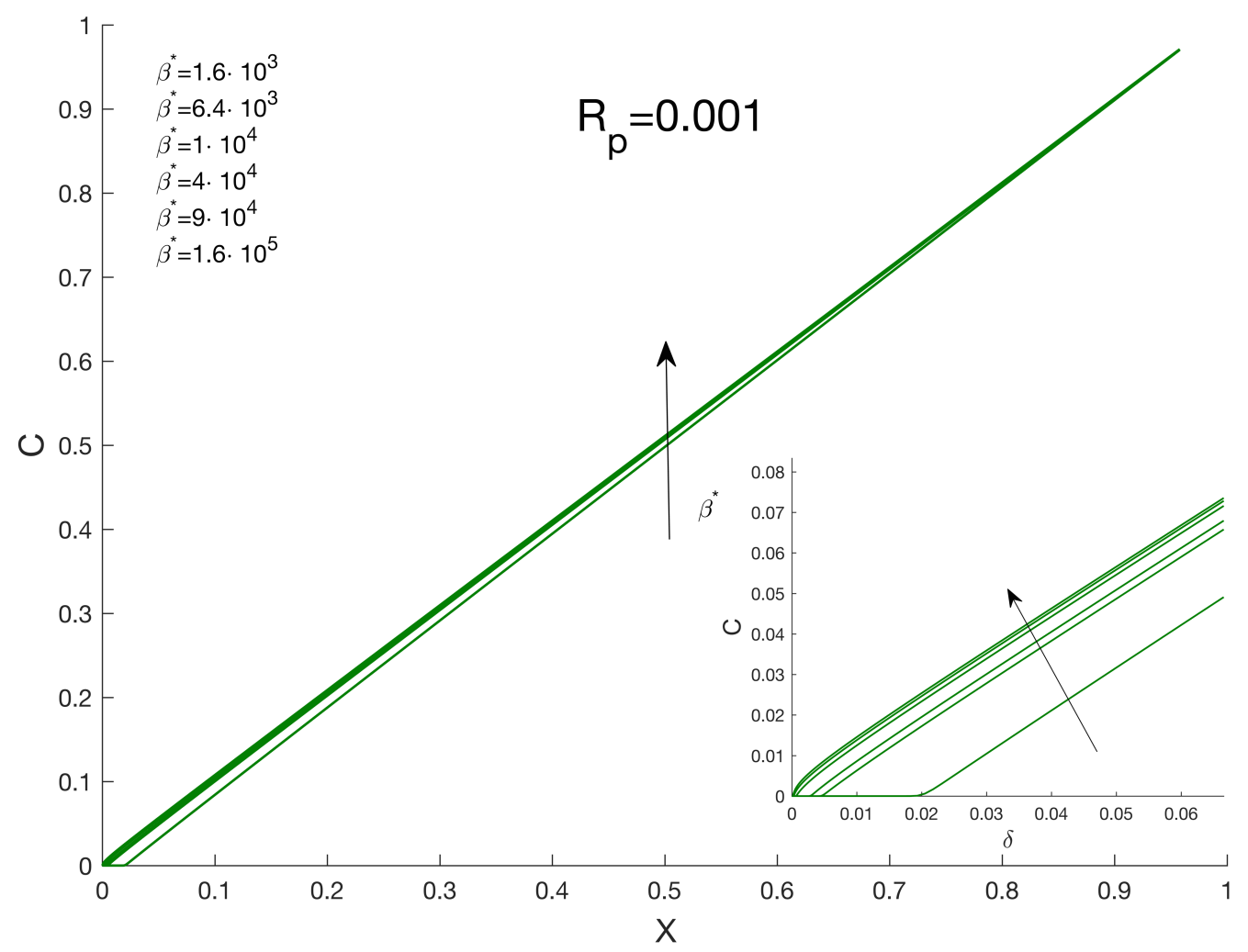

Figure 5: Concentration profiles as function of $\delta$ for various $\beta^{*} . R_{p}=0.001$. The inset shows a close view of the adsorbing layer next to the electrode. 


\section{Conclusions}

The hindered diffusion layer plays an important role in calculating flux of particles from the bulk towards an adsorbing layer next to targets. The model which is inspired by the impact of particles at electrodes, show that the smaller are the diffusion layer and adsorbing layers, the stronger is the reduction of the calculated flux from a non-hindered case. This has a direct consequences on the flux prediction of particles towards small target in different sizes, convective systems such as the rotating disc electrode, and even in the limiting case of the Nernst layer, often used as a simple model for transport in stationary solution. We have also shown that the concentration profiles are influenced throughout the diffusion layer,in the case where the radius of a particle relative to the size of the diffusion layer is non-negligible.

\section{References}

(1) Xiao, X.; Bard, A. J. Observing single nanoparticle collisions at an ultramicroelectrode by electrocatalytic amplification. J. Am. Chem. Soc. 2007, 129, 9610-9612.

(2) Rees, N. V.; Zhou, Y.-G.; Compton, R. G. Making contact: Charge Transfer During Particle-Electrode Collisions. RSC Adv. 2012, 2, 379-384.

(3) Fan, F.-R. F.; Bard, A. J. An electrochemical coulomb staircase: detection of single electron-transfer events at nanometer electrodes. Science 1997, 277, 1791-1793.

(4) Kätelhön, E.; Compton, R. G. Understanding Nano-Impacts: Binary Nature of Charge Transfer during Mediated Reactions. ChemElectroChem 2015, 2, 64-67.

(5) Bard, A. J.; Zhou, H.; Kwon, S. J. Electrochemistry of Single Nanoparticles via Electrocatalytic Amplification. Israel Journal of Chemistry 2010, 50, 267-276.

(6) Zhou, Y.-G.; Rees, N. V.; Compton, R. G. The Electrochemical Detection and Charac- 
terization of Silver Nanoparticles in Aqueous Solution. Angew. Chem., Int. Ed. 2011, 50, 4219-4221.

(7) Sardesai, N. P.; Andreescu, D.; Andreescu, S. Electroanalytical Evaluation of Antioxidant Activity of Cerium Oxide Nanoparticles by Nanoparticle Collisions at Microelectrodes. J. Am. Chem. Soc. 2013, 135, 16770-16773.

(8) Alligrant, T. M.; Nettleton, E. G.; Crooks, R. M. Electrochemical Detection of Individual DNA Hybridization Events. Lab Chip 2013, 13, 349-354.

(9) Dick, J. E.; Hilterbrand, A. T.; Boika, A.; Upton, J. W.; Bard, A. J. Electrochemical Detection of a Single Cytomegalovirus at an Ultramicroelectrode and its Antibody Anchoring. Proceedings of the National Academy of Sciences 2015, 112, 5303-5308.

(10) Cheng, W.; Compton, R. G. Investigation of Single-Drug-Encapsulating Liposomes using the Nano-Impact Method. Angew. Chem., Int. Ed. 2014, 53, 13928-13930.

(11) Sepunaru, L.; Tschulik, K.; Batchelor-McAuley, C.; Gavish, R.; Compton, R. G. Electrochemical detection of single E. coli bacteria labeled with silver nanoparticles. Biomaterials Science 2015, 3, 816-820.

(12) Pumera, M. Impact Electrochemistry: Measuring Individual Nanoparticles. ACS Nano 2014, 8, 7555-7558.

(13) Stuart, E. J. E.; Tschulik, K.; Batchelor-McAuley, C.; Compton, R. G. Electrochemical Observation of Single Collision Events: Fullerene Nanoparticles. ACS Nano 2014, 8, $7648-7654$.

(14) Edward, J. T. Molecular Volumes and the Stokes-Einstein Equation. Journal of Chemical Education 1970, 47, 261.

(15) Brenner, H. The Slow Motion of a Sphere through a Viscous Fluid towards a Plane Surface. Chemical Engineering Science 1961, 16, 242-251. 
(16) Huang, P.; Breuer, K. S. Direct measurement of anisotropic near-wall hindered diffusion using total internal reflection velocimetry. Phys. Rev. E 2007, 76, 046307.

(17) Bevan, M. A.; Prieve, D. C. Hindered Diffusion of Colloidal Particles very Near to a Wall: Revisited. J. Chem. Phys. 2000, 113, 1228-1236.

(18) Karger, J.; Ruthven, D. M. Diffusion in Zeolites and Other Microporous Solids; Wiley, New York, 1992; pp 42, 90.

(19) Dettmer, S. L.; Pagliara, S.; Misiunas, K.; Keyser, U. F. Anisotropic Diffusion of Spherical Particles in Closely Confining Microchannels. Phys. Rev. E 2014, 89, 062305.

(20) Pagliara, S.; Schwall, C.; Keyser, U. F. Optimizing diffusive transport through a synthetic membrane channel. Advanced Materials 2013, 25, 844-849.

(21) Jain, R. K.; Stylianopoulos, T. Delivering nanomedicine to solid tumors. Nature Reviews Clinical Oncology 2010, 7, 653-664.

(22) Stuart, E. J. E.; Zhou, Y.-G.; Rees, N. V.; Compton, R. G. Determining Unknown Concentrations of Nanoparticles: the Particle-impact Electrochemistry of Nickel and Silver. RSC Adv. 2012, 2, 6879-6884.

(23) Boika, A.; Bard, A. J. Time of First Arrival in Electrochemical Collision Experiments as a Measure of Ultralow Concentrations of Analytes in Solution. Analytical Chemistry 2015, 87, 4341-4346.

(24) Eloul, S.; Kätelhön, E.; Compton, R. G. When does near-wall hindered diffusion influence mass transport towards targets? Phys. Chem. Chem. Phys. 2016, In press,

(25) Compton, R. G.; Banks, C. E. Understanding voltammetry; Imperial College Press: London, UK, 2006. 
(26) Sokolov, S. V.; Kätelhön, E.; Compton, R. G. Near-wall Hindered Diffusion in Convective Systems: Transport Limitations in Colloidal and Nanoparticulate Systems. J. Phys. Chem. C 2016,

(27) Bard, A. J.; Faulkner, L. Electrochemical Methods: Fundamentals and Applications; John Wiley and Sons, 2001.

(28) Amatore, C.; Szunerits, S.; Thouin, L.; Warkocz, J.-S. The real meaning of Nernst's steady diffusion layer concept under non-forced hydrodynamic conditions. A simple model based on Levich's seminal view of convection. Journal of Electroanalytical Chemistry 2001, 500, $62-70$.

(29) Holcman, D. Modeling DNA and virus trafficking in the cell cytoplasm. Journal of Statistical Physics 2007, 127, 471-494.

(30) Singer, A.; Schuss, Z.; Holcman, D. Narrow escape and leakage of Brownian particles. Phys. Rev. E 2008, 78, 051111.

(31) Zhdanov, V. P.; Höök, F. Diffusion-limited attachment of large spherical particles to flexible membrane-immobilized receptors. European Biophysics Journal 2015, 44, 219226.

(32) Danhier, F.; Feron, O.; Préat, V. To exploit the tumor microenvironment: passive and active tumor targeting of nanocarriers for anti-cancer drug delivery. Journal of Controlled Release 2010, 148, 135-146.

(33) Hugelmann, M.; Schindler, W. Tunnel barrier height oscillations at the solid/liquid interface. Surface Science 2003, 541, L643-L648.

(34) Edwards, P. P.; Gray, H.; Lodge, M.; Williams, R. J. Electron Transfer and Electronic Conduction through an Intervening Medium. Angew. Chem., Int. Ed. 2008, 47, 67586765. 
(35) Eloul, S.; Kätelhön, E.; Batchelor-McAuley, C.; Tschulik, K.; Compton, R. G. Diffusional impacts of Nanoparticles on Microdisc and Microwire Electrodes: The Limit of Detection and First Passage Statistics. J. Electroanal. Chem. 2015, 755, 136-142.

(36) Schuss, Z.; Singer, A.; Holcman, D. The narrow escape problem for diffusion in cellular microdomains. Proceedings of the National Academy of Sciences 2007, 104, 1609816103. 